\title{
A Space-Time Fully Decoupled Wavelet Galerkin Method for Solving Multidimensional Nonlinear Schrödinger Equations with Damping
}

\author{
Jiaqun Wang, ${ }^{1}$ Youhe Zhou, ${ }^{1}$ and Xiaojing Liu ${ }^{1,2}$ \\ ${ }^{1}$ Key Laboratory of Mechanics on Disaster and Environment in Western China, The Ministry of Education, \\ College of Civil Engineering and Mechanics, Lanzhou University, Lanzhou 730000, China \\ ${ }^{2}$ State Key Laboratory of Structural Analysis for Industrial Equipment, Dalian University of Technology, Dalian 116024, China
}

Correspondence should be addressed to Xiaojing Liu; liuxiaojing@lzu.edu.cn

Received 13 July 2017; Accepted 9 November 2017; Published 28 November 2017

Academic Editor: Hang Xu

Copyright (C) 2017 Jiaqun Wang et al. This is an open access article distributed under the Creative Commons Attribution License, which permits unrestricted use, distribution, and reproduction in any medium, provided the original work is properly cited.

\begin{abstract}
On the basis of sampling approximation for a function defined on a bounded interval by combining Coiflet-type wavelet expansion and technique of boundary extension, a space-time fully decoupled formulation is proposed to solve multidimensional Schrödinger equations with generalized nonlinearities and damping. By applying a wavelet Galerkin approach for spatial discretization, nonlinear Schrödinger equations are first transformed into a system of ordinary differential equations, in which all matrices are completely independent of time and never need to be recalculated in the time integration. Then, the classical fourth-order explicit Runge-Kutta method is used to solve the resulting semidiscretization system. By studying several widely considered test problems, results demonstrate that when a relatively fine mesh is adopted, the present wavelet algorithm has a much better computational accuracy and efficiency than many existing numerical methods, due to its higher order of convergence in space which can go up to 6.
\end{abstract}

\section{Introduction}

The time-dependent Schrödinger equation with nonlinear potential functions is one of the most important mathematical models in modern science due to its wide applications in many different fields, such as nonlinear optics, plasma physics, and semiconductor industry [1-5]. The present study considers the three-dimensional generalized nonlinear Schrödinger equation (GNLSE) [6-37]

$$
\begin{aligned}
\mathrm{i} \frac{\partial \psi(\mathbf{x}, t)}{\partial t}+\alpha(t) \nabla^{2} \psi+\nu(\mathbf{x}, t) \psi+f\left(|\psi|^{2}\right) \psi & \\
+\mathrm{i} g\left(|\psi|^{2}\right) \psi+h_{1}(\mathbf{x}, t)+\mathrm{i} h_{2}(\mathbf{x}, t) & =0, \\
& \mathbf{x} \in[0,1]^{3}, t \geq 0
\end{aligned}
$$

with the initial condition

$$
\psi(\mathbf{x}, 0)=R_{1}(\mathbf{x})+\mathrm{i} R_{2}(\mathbf{x}), \quad \mathbf{x} \in[0,1]^{3}
$$

and the Dirichlet boundary condition

$$
\begin{aligned}
& \psi(0, y, z, t)=r_{01}(y, z, t)+\mathrm{i} r_{02}(y, z, t), \\
& \psi(1, y, z, t)=s_{01}(y, z, t)+\mathrm{i} s_{02}(y, z, t) \\
& \psi(x, 0, z, t)=r_{11}(x, z, t)+\mathrm{i} r_{12}(x, z, t), \\
& \psi(x, 1, z, t)=s_{11}(x, z, t)+\mathrm{i} s_{12}(x, z, t) \\
& \psi(x, y, 0, t)=r_{21}(x, y, t)+\mathrm{i} r_{22}(x, y, t), \\
& \psi(x, y, 1, t)=s_{21}(x, y, t)+\mathrm{i} s_{22}(x, y, t) .
\end{aligned}
$$

In (1)-(3), $\mathrm{i}=\sqrt{-1}$ is the complex unit, $\psi(\mathbf{x}, t)$ with vector $\mathbf{x}=\{x, y, z\}$ is a complex-valued unknown function, $\nabla^{2}=$ $\partial^{2} / \partial x^{2}+\partial^{2} / \partial y^{2}+\partial^{2} / \partial z^{2}$ is the Laplace operator, and $\alpha(t)$, $R_{j}, r_{i j}$, and $s_{i j}$ are known real functions for $i=0,1,2$ and $j=1,2$. In GNLSE (1), the external potential $\nu(\mathbf{x}, t)$ is a given real function, whose specific form is dependent on different problems. For example, it is usually chosen as 
either a harmonic confining potential $v(\mathbf{x}, t)=-|\mathbf{x}|^{2} / 2$, or an optical lattice potential $v(\mathbf{x}, t)=A_{1} \cos \left(L_{1} x\right)+$ $A_{2} \cos \left(L_{2} y\right)+A_{3} \cos \left(L_{3} z\right)$ with constants $A_{n}, L_{n}$, and $n=$ $1,2,3$, for studying the Bose-Einstein condensation $[5,26]$. The nonlinearities $f(\rho)$ and $g(\rho)$ which are real-valued smooth functions with respect to the density $\rho=|\psi|^{2}$ are determined by the specific application. Note that, in this study, the most popular nonlinear term $f(\rho)=\lambda \rho$ with constant $\lambda[1-10]$ has been extended to the general form $f(\rho)$ which can be chosen as arbitrary function as needed, such as $f(\rho)=c_{0} \rho^{s}, f(\rho)=c_{1}\left(1-e^{-\rho}\right), f(\rho)=\rho /(1+\rho)$, and $f(\rho)=\ln (1+\rho)$, where $c_{0}$, $s$ and $c_{1}$ are real constants $[26,36]$. Moreover, the general damping term $g(\rho)$ which is usually ignored in many studies [8-16, 18-25, 27-34] is also considered in GNLSE (1), because the damping effect may play an important role and should not be ignored in some physical processes $[7,17,26,35,36]$, such as the inelastic collisions in Bose-Einstein condensation $[7,26]$.

Because of the broad applications of GNLSE (1), developing accurate and efficient numerical methods for solving such equations has attracted considerable research attention in the past few years. For instance, Mohebbi and Dehghan [15] studied two-dimensional linear Schrödinger equations by using a compact boundary value method with fourth-order accuracy in both space and time. Liao et al. [16] applied a fourth-order compact difference scheme to solve the two-dimensional linear Schrödinger equation without damping term. In order to save cost on computation, the finite difference schemes combining with the alternating direction implicit method are developed to study the multidimensional Schrödinger equations [18-20, 23, 24, 30, 34]. In such algorithms, handling multidimensional problems is transformed into solving a series of one-dimensional problems by introducing intermediate variables. Moreover, many other numerical methods are also proposed to solve multidimensional Schrödinger equations, such as the collocation method [21, 22, 27, 33], the Galerkin method [27, 31], and the mesh-free methods $[25,31,32]$. The above methods are effective for solving Schrödinger equations under certain conditions. However, many of them will encounter severe difficulties in uniformly solving the three-dimensional generalized nonlinear Schrödinger equation (1). For example, the time-splitting methods need to obtain the density $\rho$ by solving analytically a nonlinear differential equation $[7,8,21,26]$, which is an extremely difficult task for general damping term $g(\rho)$. And when the classical collocation method [25] and Galerkin type method [32] are employed to solve directly the nonlinear Schrödinger equation, the matrices generated in the spatial discretization of nonlinear terms will be dependent on the time-dependent unknown vector and must be recalculated at each time step $[25,32,38]$, thereby consuming considerable computing resources. Because such repeated recalculations of matrices from the spatial discretization of nonlinear term can be regarded as reperforming the spatial discretization at each time step [38], these methods [25, 27, 32] cannot divide the solution procedure into two completely separate processes, that is, the spatial discretization and the time integration. Therefore, the decoupling between spatial and temporal discretizations in these methods $[25,27,32]$ is incomplete [38].

In the current work, a space-time fully decoupled formulation by combining a wavelet Galerkin technique and the classical fourth-order explicit Runge-Kutta method is proposed to uniformly solve the three-dimensional generalized nonlinear Schrödinger equation (1). In such a space-time fully decoupled wavelet formulation, all matrices generated in the spatial discretization of the nonlinear partial differential equation (1) are constant matrices and need not to be updated in the subsequent time integration. In addition, a systematic comparison between the present solutions and those obtained by using many existing numerical methods is conducted by solving several widely considered test problems.

\section{Sampling Approximation of an Interval-Bounded $L^{2}$-Function}

Based on the theory of wavelet based multiresolution analysis, a set of scaling bases for three-dimensional space can be directly obtained by the tensor products of one-dimensional wavelet bases $[39,40]$. Therefore following our previous work [41-44], a function $f(x, y, z) \in L^{2}[0,1]^{3}$ can be approximated as

$$
\begin{aligned}
& f(x, y, z) \approx P^{j_{x}, j_{y}, j_{z}} f(x, y, z) \\
& =\sum_{k_{0}=0}^{2^{j_{x}}} \sum_{l_{0}=0}^{2^{j_{y}}} \sum_{m_{0}=0}^{2^{j_{z}}} f\left(\frac{k_{0}}{2^{j_{x}}}, \frac{l_{0}}{2^{j_{y}}}, \frac{m_{0}}{2^{j_{z}}}\right) \varphi_{j_{x}, k_{0}}(x) \varphi_{j_{y}, l_{0}}(y) \\
& \cdot \varphi_{j_{z}, m_{0}}(z)
\end{aligned}
$$

where $j_{x}, j_{y}$, and $j_{z}$ are the decomposition level, respectively, in the $x, y$, and $z$ directions, and the modified onedimensional wavelet basis [41-44]

$$
\varphi_{j, k}(x)= \begin{cases}\sum_{i=-9}^{-1} T_{0, k}\left(\frac{i}{2^{j}}\right) \phi\left(2^{j} x-i+7\right)+\phi\left(2^{j} x-k+7\right) & k \in[0, M] \\ \phi\left(2^{j} x-k+7\right) & k \in\left[M+1,2^{j}-M-1\right] \\ \sum_{i=2^{j}+1}^{2^{j}+6} T_{1,2^{j}-k}\left(\frac{i}{2^{j}}\right) \phi\left(2^{j} x-i+7\right)+\phi\left(2^{j} x-k+7\right) & k \in\left[2^{j}-M, 2^{j}\right]\end{cases}
$$


with

$$
M= \begin{cases}2^{j-1}-1 & \text { for } j \leq 3 \\ 7 & \text { other. }\end{cases}
$$

In (5), $\phi(x)$ is the generalized Coiflet-type orthogonal scaling function with first-order moment $M_{1}=7$ and number of vanishing moments $\beta=6$ of the corresponding wavelet function, which is developed by Wang [41]. In addition, the functions

$$
\begin{aligned}
T_{0, k}(x) & =\sum_{i=0}^{M} \frac{p_{0, i, k}}{i !} x^{i}, \\
T_{1, k} & =\sum_{i=0}^{M} \frac{p_{1, i, k}}{i !}(x-1)^{i}
\end{aligned}
$$

in which coefficients $p_{0, i, k}$ and $p_{1, i, k}$ of numerical differentiation are determined by matrices $\mathbf{P}_{0}=\left\{p_{0, i, k}\right\}=\mathbf{Q}_{0}^{-1}, \mathbf{P}_{1}=$ $\left\{p_{1, i, k}\right\}=\mathbf{Q}_{1}^{-1}, \mathbf{Q}_{0}=\left\{q_{0, k, i}=\left(k / 2^{j}\right)^{i} / i !\right\}$, and $\mathbf{Q}_{1}=\left\{q_{1, k, i}=\right.$ $\left(-k / 2^{j}\right)^{i} / i$ ! \} with subscripts $i, k=0,1, \ldots, M$.

Since the sampling approximation (4) is valid for all functions $f(x, y, z) \in L^{2}[0,1]^{3}$, for arbitrary nonlinear operator $\mathbf{N}$ holding $\mathbf{N}[f(x, y, z)] \in L^{2}[0,1]^{3}$, by treating $\mathbf{N}[f(x, y, z)]$ as a new function and applying $(4)$, we have

$$
\begin{gathered}
\mathbf{N}[f(x, y, z)] \approx \sum_{k_{0}=0}^{2^{j_{x}}} \sum_{l_{0}=0}^{2^{j_{y}}} \sum_{m_{0}=0}^{2^{j_{z}}} \mathbf{N}\left[f\left(\frac{k_{0}}{2^{j_{x}}}, \frac{l_{0}}{2^{j_{y}}}, \frac{m_{0}}{2^{j_{z}}}\right)\right] \\
\cdot \varphi_{j_{x}, k_{0}}(x) \varphi_{j_{y}, l_{0}}(y) \varphi_{j_{z}, m_{0}}(z)
\end{gathered}
$$

which provides the foundation for conducting a space-time fully decoupled formulation [38].

\section{Solution of the Generalized Nonlinear Schrödinger Equation}

At the beginning of the solution to the three-dimensional generalized nonlinear Schrödinger equation (1), by introducing $\psi(\mathbf{x}, t)=u_{1}(\mathbf{x}, t)+\mathrm{i} u_{2}(\mathbf{x}, t)$, where $u_{1}(\mathbf{x}, t)$ and $u_{2}(\mathbf{x}, t)$ are real-valued functions, we rewrite it into two coupled timedependent nonlinear partial differential equations

$$
\begin{aligned}
\frac{\partial u_{n}(\mathbf{x}, t)}{\partial t}= & (-1)^{n} \alpha(t) \nabla^{2} u_{m}+(-1)^{n} \nu(\mathbf{x}, t) u_{m} \\
& +(-1)^{n} f\left(u_{n}^{2}+u_{m}^{2}\right) u_{m}+(-1)^{n} h_{m}(\mathbf{x}, t) \\
& -g\left(u_{n}^{2}+u_{m}^{2}\right) u_{n},
\end{aligned}
$$

in which $n=1,2$ and $m=3-n$.

Following the wavelet approximations (4) and (8), the unknown functions $u_{n}(\mathbf{x}, t)$, nonlinear terms $f\left(u_{n}^{2}+\right.$ $\left.u_{m}^{2}\right) u_{m}(\mathbf{x}, t)$ and $g\left(u_{n}^{2}+u_{m}^{2}\right) u_{n}(\mathbf{x}, t)$, and terms $\nu(\mathbf{x}, t) u_{m}(\mathbf{x}, t)$ and $h_{m}(\mathbf{x}, t)$ can be expressed as

$$
\begin{aligned}
& u_{n}(\mathbf{x}, t) \approx \sum_{k_{0}=0}^{2^{j_{x}}} \sum_{l_{0}=0}^{2^{j_{y}}} \sum_{m_{0}=0}^{2^{j_{z}}} u_{n}\left(\frac{k_{0}}{2^{j_{x}}}, \frac{l_{0}}{2^{j_{y}}}, \frac{m_{0}}{2^{j_{z}}}, t\right) \varphi_{j_{x}, k_{0}}(x) \varphi_{j_{y}, l_{0}}(y) \varphi_{j_{z}, m_{0}}(z), \\
& v(\mathbf{x}, t) u_{m}(\mathbf{x}, t) \approx \sum_{k_{0}=0}^{2^{j_{x}}} \sum_{l_{0}=0}^{2^{j_{y}}} \sum_{m_{0}=0}^{2^{j_{z}}} v\left(\frac{k_{0}}{2^{j_{x}}}, \frac{l_{0}}{2^{j_{y}}}, \frac{m_{0}}{2^{j_{z}}}, t\right) u_{m}\left(\frac{k_{0}}{2^{j_{x}}}, \frac{l_{0}}{2^{j_{y}}}, \frac{m_{0}}{2^{j_{z}}}, t\right) \varphi_{j_{x}, k_{0}}(x) \varphi_{j_{y}, l_{0}}(y) \varphi_{j_{z}, m_{0}}(z), \\
& f\left(u_{n}^{2}+u_{m}^{2}\right) u_{m}(\mathbf{x}, t) \approx \sum_{k_{0}=0}^{2^{j_{x}}} \sum_{l_{0}=0}^{2^{j_{y}}} \sum_{m_{0}=0}^{2^{j_{z}}} f\left[u_{n}^{2}\left(\frac{k_{0}}{2^{j_{x}}}, \frac{l_{0}}{2^{j_{y}}}, \frac{m_{0}}{2^{j_{z}}}, t\right)+u_{m}^{2}\left(\frac{k_{0}}{2^{j_{x}}}, \frac{l_{0}}{2^{j_{y}}}, \frac{m_{0}}{2^{j_{z}}}, t\right)\right] \\
& \times u_{m}\left(\frac{k_{0}}{2^{j_{x}}}, \frac{l_{0}}{2^{j_{y}}}, \frac{m_{0}}{2^{j_{z}}}, t\right) \varphi_{j_{x}, k_{0}}(x) \varphi_{j_{y}, l_{0}}(y) \varphi_{j_{z}, m_{0}}(z), \\
& g\left(u_{n}^{2}+u_{m}^{2}\right) u_{n}(\mathbf{x}, t) \approx \sum_{k_{0}=0}^{2^{j_{x}}} \sum_{l_{0}=0}^{2^{j_{y}}} \sum_{m_{0}=0}^{2^{j_{z}}} g\left[u_{n}^{2}\left(\frac{k_{0}}{2^{j_{x}}}, \frac{l_{0}}{2^{j_{y}}}, \frac{m_{0}}{2^{j_{z}}}, t\right)+u_{m}^{2}\left(\frac{k_{0}}{2^{j_{x}}}, \frac{l_{0}}{2^{j_{y}}}, \frac{m_{0}}{2^{j_{z}}}, t\right)\right] \\
& \times u_{n}\left(\frac{k_{0}}{2^{j_{x}}}, \frac{l_{0}}{2^{j_{y}}}, \frac{m_{0}}{2^{j_{z}}}, t\right) \varphi_{j_{x}, k_{0}}(x) \varphi_{j_{y}, l_{0}}(y) \varphi_{j_{z}, m_{0}}(z), \\
& h_{m}(\mathbf{x}, t) \approx \sum_{k_{0}=0}^{2^{j_{x}}} \sum_{l_{0}=0}^{2^{j_{y}}} \sum_{m_{0}=0}^{2^{j_{z}}} h_{m}\left(\frac{k_{0}}{2^{j_{x}}}, \frac{l_{0}}{2^{j_{y}}}, \frac{m_{0}}{2^{j_{z}}}, t\right) \varphi_{j_{x}, k_{0}}(x) \varphi_{j_{y}, l_{0}}(y) \varphi_{j_{z}, m_{0}}(z) .
\end{aligned}
$$

Substituting (10)-(14) into (9), then multiplying both sides of the resulting equation by $\varphi_{j_{x}, k_{1}}(x) \varphi_{j_{y}, l_{1}}(y) \varphi_{j_{z}, m_{1}}(z)$, $k_{1}=1,2, \ldots, 2^{j_{x}}-1, l_{1}=1,2, \ldots, 2^{j_{y}}-1$, and $m_{1}=$ $1,2, \ldots, 2^{j_{z}}-1$, respectively, and performing integration 
over the domain $[0,1]^{3}$, one can obtain a series of ordinary differential equations. Considering boundary conditions (3), these ordinary differential equations can be written into

$$
\begin{aligned}
\frac{d \mathbf{U}(t)}{d t}= & \frac{d}{d t}\left[\begin{array}{l}
\mathbf{U}_{1}(t) \\
\mathbf{U}_{2}(t)
\end{array}\right] \approx \mathbf{G}[\mathbf{U}(t), t] \\
= & {\left[\begin{array}{l}
\mathbf{G}_{1}\left[\mathbf{U}_{1}(t), \mathbf{U}_{2}(t), t\right] \\
\mathbf{G}_{2}\left[\mathbf{U}_{1}(t), \mathbf{U}_{2}(t), t\right]
\end{array}\right], } \\
\mathbf{G}_{n}= & (-1)^{n} \alpha(t) \mathbf{A}^{-1} \mathbf{B} \mathbf{U}_{m}+(-1)^{n} \mathbf{V} * \mathbf{U}_{m} \\
& +(-1)^{n} f\left(\mathbf{U}_{n} * \mathbf{U}_{n}+\mathbf{U}_{m} * \mathbf{U}_{m}\right) * \mathbf{U}_{m} \\
& +(-1)^{n} \mathbf{H}_{m}-g\left(\mathbf{U}_{n} * \mathbf{U}_{n}+\mathbf{U}_{m} * \mathbf{U}_{m}\right) \\
& * \mathbf{U}_{n}+\mathbf{A}^{-1} \mathbf{C}_{n}(t)
\end{aligned}
$$

in which vectors $\mathbf{U}_{n}(t)=\left\{u_{n, p}(t)=u_{n}\left(k_{0} / 2^{j_{x}}, l_{0} / 2^{j_{y}}, m_{0} / 2^{j_{z}}\right.\right.$, $t)\}^{\mathrm{T}}, \mathbf{V}(t)=\left\{v_{p}(t)=v\left(k_{0} / 2^{j_{x}}, l_{0} / 2^{j_{y}}, m_{0} / 2^{j_{z}}, t\right)\right\}^{\mathrm{T}}, \mathbf{H}_{m}(t)=$ $\left\{h_{m, p}(t)=h_{m}\left(k_{0} / 2^{j_{x}}, l_{0} / 2^{j_{y}}, m_{0} / 2^{j_{z}}, t\right)\right\}^{\mathrm{T}}$, matrices $\mathbf{B}=\left\{b_{o p}=\right.$ $\left.\Gamma_{k_{1}, k_{0}}^{j, 2} \Gamma_{l_{1}, l_{0}}^{j, 0} \Gamma_{m_{1} m_{0}}^{j, 0}+\Gamma_{k_{1}, k_{0}}^{j, 0} \Gamma_{l_{1}, l_{0}}^{j, 2} \Gamma_{m_{1} m_{0}}^{j, 0}+\Gamma_{k_{1}, k_{0}}^{j, 0} \Gamma_{l_{1}, l_{0}}^{j, 0} \Gamma_{m_{1} m_{0}}^{j, 2}\right\}, \mathbf{A}=\left\{a_{o p}=\right.$ $\left.\Gamma_{k_{1}, k_{0}}^{j, 0} \Gamma_{l_{1}, l_{0}}^{j, 0} \Gamma_{m_{1} m_{0}}^{j, 0}\right\}$, and subscripts $k_{0}, k_{1}=1,2, \ldots, 2^{j_{x}}-1, l_{0}, l_{1}=$ $1,2, \ldots, 2^{j_{y}}-1, m_{0}, m_{1}=1,2, \ldots, 2^{j_{z}}-1, o=\left(2^{j_{z}}-1\right)\left[\left(2^{j_{y}}-\right.\right.$ 1) $\left.\left(k_{1}-1\right)+l_{1}-1\right]+m_{1}, p=\left(2^{j_{z}}-1\right)\left[\left(2^{j_{y}}-1\right)\left(k_{0}-1\right)+l_{0}-\right.$ 1] $+m_{0}, n=1,2$, and $m=3-n$. In (15) and (16), vector operational rules $\mathbf{P} * \mathbf{Q}=\left\{p_{k} q_{k}\right\}^{\mathrm{T}}$ and $f(\mathbf{P})=\left\{f\left(p_{k}\right)\right\}^{\mathrm{T}}$ will always hold for vectors $\mathbf{P}=\left\{p_{k}\right\}^{\mathrm{T}}$ and $\mathbf{Q}=\left\{q_{k}\right\}^{\mathrm{T}}$, and the generalized connection coefficient $\Gamma_{k_{1}, k}^{j, \alpha}=\int_{0}^{1} d^{\alpha} \varphi_{j, k}(x) / d x^{\alpha} \varphi_{j, k_{1}}(x) d x$ can be exactly and readily obtained without numerical integral but based on the database independent of the specific problems [38]. In addition, the vector $\mathbf{C}_{n}(t)$ determined by boundary conditions can be expressed as

$$
\begin{aligned}
\mathbf{C}_{n}(t) & =\left\{c_{n, o}\right. \\
= & \sum_{l_{0}=0}^{2^{j_{y}}} \sum_{m_{0}=0}^{2^{j_{z}}}\left[\sigma_{n, o, 1}\left(l_{0}, m_{0}, t\right)+\omega_{n, o, 1}\left(l_{0}, m_{0}, t\right)\right] \\
& +\sum_{k_{0}=1}^{2^{j_{x}}-1} \sum_{m_{0}=1}^{2_{z}-1}\left[\sigma_{n, o, 2}\left(k_{0}, m_{0}, t\right)+\omega_{n, o, 2}\left(k_{0}, m_{0}, t\right)\right] \\
& \left.+\sum_{k_{0}=1}^{2^{j_{x}}-1} \sum_{2_{0}=1}^{2_{y}-1}\left[\sigma_{n, o, 3}\left(k_{0}, l_{0}, t\right)+\omega_{n, 0,3}\left(k_{0}, l_{0}, t\right)\right]\right\}^{\mathrm{T}}
\end{aligned}
$$

with

$$
\begin{aligned}
& \sigma_{n, o, 1}\left(l_{0}, m_{0}, t\right)=(-1)^{n} \alpha(t) r_{0 n}\left(\frac{l_{0}}{2^{j_{y}}}, \frac{m_{0}}{2^{j_{z}}}, t\right) \\
& \cdot\left(\Gamma_{k_{1}, 0}^{j, 2} \Gamma_{l_{1}, l_{0}}^{j, 0} \Gamma_{m_{1}, m_{0}}^{j, 0}+\Gamma_{k_{1}, 0}^{j, 0} \Gamma_{l_{1}, l_{0}}^{j, 2} \Gamma_{m_{1}, m_{0}}^{j, 0}+\Gamma_{k_{1}, 0}^{j, 0} \Gamma_{l_{1}, l_{0}}^{j, 0} \Gamma_{m_{1}, m_{0}}^{j, 2}\right)
\end{aligned}
$$

$$
\begin{aligned}
& +\Gamma_{k_{1}, 0}^{j, 0} \Gamma_{l_{1}, l_{0}}^{j, 0} \Gamma_{m_{1}, m_{0}}^{j, 0}\left\{(-1)^{n} \nu\left(0, \frac{l_{0}}{2^{j_{y}}}, \frac{m_{0}}{2^{j_{z}}}, t\right)\right. \\
& \cdot r_{0 m}\left(\frac{l_{0}}{2^{j_{y}}}, \frac{m_{0}}{2^{j_{z}}}, t\right)-\frac{d r_{0 n}\left(l_{0} / 2^{j_{y}}, m_{0} / 2^{j_{z}}, t\right)}{d t} \\
& +(-1)^{n} f\left[r_{0 n}^{2}\left(\frac{l_{0}}{2^{j_{y}}}, \frac{m_{0}}{2^{j_{z}}}, t\right)+r_{0 m}^{2}\left(\frac{l_{0}}{2^{j_{y}}}, \frac{m_{0}}{2^{j_{z}}}, t\right)\right] \\
& \cdot r_{0 m}\left(\frac{l_{0}}{2^{j_{y}}}, \frac{m_{0}}{2^{j_{z}}}, t\right) \\
& -g\left[r_{0 n}^{2}\left(\frac{l_{0}}{2^{j_{y}}}, \frac{m_{0}}{2^{j_{z}}}, t\right)+r_{0 m}^{2}\left(\frac{l_{0}}{2^{j_{y}}}, \frac{m_{0}}{2^{j_{z}}}, t\right)\right] \\
& \left.\cdot r_{0 n}\left(\frac{l_{0}}{2^{j_{y}}}, \frac{m_{0}}{2^{j_{z}}}, t\right)+(-1)^{n} h_{m}\left(0, \frac{l_{0}}{2^{j_{y}}}, \frac{m_{0}}{2^{j_{z}}}, t\right)\right\},
\end{aligned}
$$$$
\omega_{n, o, 1}\left(l_{0}, m_{0}, t\right)=(-1)^{n} \alpha(t) s_{0 n}\left(\frac{l_{0}}{2^{j_{y}}}, \frac{m_{0}}{2^{j_{z}}}, t\right)
$$$$
\cdot\left(\Gamma_{k_{1}, 2^{j} x}^{j, 2} \Gamma_{l_{1}, l_{0}}^{j, 0} \Gamma_{m_{1}, m_{0}}^{j, 0}+\Gamma_{k_{1}, 2^{j / x}}^{j, 0} \Gamma_{l_{1}, l_{0}}^{j, 2} \Gamma_{m_{1}, m_{0}}^{j, 0}\right.
$$$$
\left.+\Gamma_{k_{1}, 2 j_{x}}^{j, 0} \Gamma_{l_{1}, l_{0}}^{j, 0} \Gamma_{m_{1}, m_{0}}^{j, 2}\right)+\Gamma_{k_{1}, 2{ }^{j x}}^{j, 0} \Gamma_{l_{1}, l_{0}}^{j, 0} \Gamma_{m_{1}, m_{0}}^{j, 0}\left\{(-1)^{n}\right.
$$$$
\cdot v\left(1, \frac{l_{0}}{2^{j_{y}}}, \frac{m_{0}}{2^{j_{z}}}, t\right) s_{0 m}\left(\frac{l_{0}}{2^{j_{y}}}, \frac{m_{0}}{2^{j_{z}}}, t\right)
$$$$
-\frac{d s_{0 n}\left(l_{0} / 2^{j_{y}}, m_{0} / 2^{j_{z}}, t\right)}{d t}+(-1)^{n}
$$$$
\cdot f\left[s_{0 n}^{2}\left(\frac{l_{0}}{2^{j_{y}}}, \frac{m_{0}}{2^{j_{z}}}, t\right)+s_{0 m}^{2}\left(\frac{l_{0}}{2^{j_{y}}}, \frac{m_{0}}{2^{j_{z}}}, t\right)\right]
$$$$
\cdot s_{0 m}\left(\frac{l_{0}}{2^{j_{y}}}, \frac{m_{0}}{2^{j_{z}}}, t\right)
$$$$
-g\left[s_{0 n}^{2}\left(\frac{l_{0}}{2^{j_{y}}}, \frac{m_{0}}{2^{j_{z}}}, t\right)+s_{0 n}^{m}\left(\frac{l_{0}}{2^{j_{y}}}, \frac{m_{0}}{2^{j_{z}}}, t\right)\right]
$$$$
\left.\cdot s_{0 n}\left(\frac{l_{0}}{2^{j_{y}}}, \frac{m_{0}}{2^{j_{z}}}, t\right)+(-1)^{n} h_{m}\left(1, \frac{l_{0}}{2^{j_{y}}}, \frac{m_{0}}{2^{j_{z}}}, t\right)\right\} \text {, }
$$$$
\sigma_{n, 0,2}\left(k_{0}, m_{0}, t\right)=(-1)^{n} \alpha(t) r_{1 n}\left(\frac{k_{0}}{2^{j_{x}}}, \frac{m_{0}}{2^{j_{z}}}, t\right)
$$$$
\cdot\left(\Gamma_{k_{1}, k_{0}}^{j, 2} \Gamma_{l_{1}, 0}^{j, 0} \Gamma_{m_{1}, m_{0}}^{j, 0}+\Gamma_{k_{1}, k_{0}}^{j, 0} \Gamma_{l_{1}, 0}^{j, 2} \Gamma_{m_{1}, m_{0}}^{j, 0}\right.
$$$$
\left.+\Gamma_{k_{1}, k_{0}}^{j, 0} \Gamma_{l_{1}, 0}^{j, 0} \Gamma_{m_{1}, m_{0}}^{j, 2}\right)+\Gamma_{k_{1}, k_{0}}^{j, 0} \Gamma_{l_{1}, 0}^{j, 0} \Gamma_{m_{1}, m_{0}}^{j, 0}\left\{(-1)^{n}\right.
$$$$
\cdot v\left(\frac{k_{0}}{2^{j_{x}}}, 0, \frac{m_{0}}{2^{j_{z}}}, t\right) r_{1 m}\left(\frac{k_{0}}{2^{j_{x}}}, \frac{m_{0}}{2^{j_{z}}}, t\right)
$$$$
-\frac{d r_{1 n}\left(k_{0} / 2^{j_{x}}, m_{0} / 2^{j_{z}}, t\right)}{d t}+(-1)^{n}
$$ 


$$
\begin{aligned}
& \cdot f\left[r_{1 n}^{2}\left(\frac{k_{0}}{2^{j_{x}}}, \frac{m_{0}}{2^{j_{z}}}, t\right)+r_{1 m}^{2}\left(\frac{k_{0}}{2^{j_{x}}}, \frac{m_{0}}{2^{j_{z}}}, t\right)\right] \\
& \cdot r_{1 m}\left(\frac{k_{0}}{2^{j_{x}}}, \frac{m_{0}}{2^{j_{z}}}, t\right) \\
& -g\left[r_{1 n}^{2}\left(\frac{k_{0}}{2^{j_{x}}}, \frac{m_{0}}{2^{j_{z}}}, t\right)+r_{1 m}^{2}\left(\frac{k_{0}}{2^{j_{x}}}, \frac{m_{0}}{2^{j_{z}}}, t\right)\right] \\
& \left.\cdot r_{1 n}\left(\frac{k_{0}}{2^{j_{x}}}, \frac{m_{0}}{2^{j_{z}}}, t\right)+(-1)^{n} h_{m}\left(\frac{k_{0}}{2^{j_{x}}}, 0, \frac{m_{0}}{2^{j_{z}}}, t\right)\right\} \text {, } \\
& \omega_{n, o, 2}\left(k_{0}, m_{0}, t\right)=(-1)^{n} \alpha(t) s_{1 n}\left(\frac{k_{0}}{2^{j_{x}}}, \frac{m_{0}}{2^{j_{z}}}, t\right) \\
& \cdot\left(\Gamma_{k_{1}, k_{0}}^{j, 2} \Gamma_{l_{1}, 2^{j y}}^{j, 0} \Gamma_{m_{1}, m_{0}}^{j, 0}+\Gamma_{k_{1}, k_{0}}^{j, 0} \Gamma_{l_{1}, 2^{j y}}^{j, 2} \Gamma_{m_{1}, m_{0}}^{j, 0}\right. \\
& \left.+\Gamma_{k_{1}, k_{0}}^{j, 0} \Gamma_{l_{1}, 2^{j y}}^{j, 0} \Gamma_{m_{1}, m_{0}}^{j, 2}\right)+\Gamma_{k_{1}, k_{0}}^{j, 0} \Gamma_{l_{1}, 2^{2} j_{y}}^{j, 0} \Gamma_{m_{1}, m_{0}}^{j, 0}\left\{(-1)^{n}\right. \\
& \cdot v\left(\frac{k_{0}}{2^{j_{x}}}, 1, \frac{m_{0}}{2^{j_{z}}}, t\right) s_{1 m}\left(\frac{k_{0}}{2^{j_{x}}}, \frac{m_{0}}{2^{j_{z}}}, t\right) \\
& -\frac{d s_{1 n}\left(k_{0} / 2^{j_{x}}, m_{0} / 2^{j_{z}}, t\right)}{d t}+(-1)^{n} \\
& \cdot f\left[s_{1 n}^{2}\left(\frac{k_{0}}{2^{j_{x}}}, \frac{m_{0}}{2^{j_{z}}}, t\right)+s_{1 m}^{2}\left(\frac{k_{0}}{2^{j_{x}}}, \frac{m_{0}}{2^{j_{z}}}, t\right)\right] \\
& \cdot s_{1 m}\left(\frac{k_{0}}{2^{j_{x}}}, \frac{m_{0}}{2^{j_{z}}}, t\right) \\
& -g\left[s_{1 n}^{2}\left(\frac{k_{0}}{2^{j_{x}}}, \frac{m_{0}}{2^{j_{z}}}, t\right)+s_{1 m}^{2}\left(\frac{k_{0}}{2^{j_{x}}}, \frac{m_{0}}{2^{j_{z}}}, t\right)\right] \\
& \left.\cdot s_{1 n}\left(\frac{k_{0}}{2^{j_{x}}}, \frac{m_{0}}{2^{j_{z}}}, t\right)+(-1)^{n} h_{m}\left(\frac{k_{0}}{2^{j_{x}}}, 1, \frac{m_{0}}{2^{j_{z}}}, t\right)\right\} \text {, } \\
& \sigma_{n, o, 3}\left(k_{0}, l_{0}, t\right)=(-1)^{n} \alpha(t) r_{2 n}\left(\frac{k_{0}}{2^{j_{x}}}, \frac{l_{0}}{2^{j_{y}}}, t\right) \\
& \cdot\left(\Gamma_{k_{1}, k_{0}}^{j, 2} \Gamma_{l_{1}, l_{0}}^{j, 0} \Gamma_{m_{1}, 0}^{j, 0}\right. \\
& \left.+\Gamma_{k_{1}, k_{0}}^{j, 0} \Gamma_{l_{1}, l_{0}}^{j, 2} \Gamma_{m_{1}, 0}^{j, 0}+\Gamma_{k_{1}, k_{0}}^{j, 0} \Gamma_{l_{1}, l_{0}}^{j, 0} \Gamma_{m_{1}, 0}^{j, 2}\right) \\
& +\Gamma_{k_{1}, k_{0}}^{j, 0} \Gamma_{l_{1}, l_{0}}^{j, 0} \Gamma_{m_{1}, 0}^{j, 0}\left\{(-1)^{n} \nu\left(\frac{k_{0}}{2^{j_{x}}}, \frac{l_{0}}{2^{j_{y}}}, 0, t\right)\right. \\
& \cdot r_{2 m}\left(\frac{k_{0}}{2^{j_{x}}}, \frac{l_{0}}{2^{j_{y}}}, t\right)-\frac{d r_{2 n}\left(k_{0} / 2^{j_{x}}, l_{0} / 2^{j_{y}}, t\right)}{d t} \\
& +(-1)^{n} f\left[r_{2 n}^{2}\left(\frac{k_{0}}{2^{j_{x}}}, \frac{l_{0}}{2^{j_{y}}}, t\right)+r_{2 m}^{2}\left(\frac{k_{0}}{2^{j_{x}}}, \frac{l_{0}}{2^{j_{y}}}, t\right)\right] \\
& \cdot r_{2 m}\left(\frac{k_{0}}{2^{j_{x}}}, \frac{l_{0}}{2^{j_{y}}}, t\right) \\
& -g\left[r_{2 n}^{2}\left(\frac{k_{0}}{2^{j_{x}}}, \frac{l_{0}}{2^{j_{y}}}, t\right)+r_{2 m}^{2}\left(\frac{k_{0}}{2^{j_{x}}}, \frac{l_{0}}{2^{j_{y}}}, t\right)\right] \\
& \left.\cdot r_{2 n}\left(\frac{k_{0}}{2^{j_{x}}}, \frac{l_{0}}{2^{j_{y}}}, t\right)+(-1)^{n} h_{m}\left(\frac{k_{0}}{2^{j_{x}}}, \frac{l_{0}}{2^{j_{y}}}, 0, t\right)\right\} \text {, }
\end{aligned}
$$

$$
\begin{aligned}
& \omega_{n, o, 3}\left(k_{0}, l_{0}, t\right)=(-1)^{n} \alpha(t) s_{2 n}\left(\frac{k_{0}}{2^{j_{x}}}, \frac{l_{0}}{2^{j_{y}}}, t\right) \\
& \cdot\left(\Gamma_{k_{1}, k_{0}}^{j, 2} \Gamma_{l_{1}, l_{0}}^{j, 0} \Gamma_{m_{1}, 2^{j z}}^{j, 0}+\Gamma_{k_{1}, k_{0}}^{j, 0} \Gamma_{l_{1}, l_{0}}^{j, 2} \Gamma_{m_{1}, 2^{j z}}^{j, 0}\right. \\
& \left.+\Gamma_{k_{1}, k_{0}}^{j, 0} \Gamma_{l_{1}, l_{0}}^{j, 0} \Gamma_{m_{1}, 2^{j z}}^{j, 2}\right)+\Gamma_{k_{1}, k_{0}}^{j, 0} \Gamma_{l_{1}, l_{0}}^{j, 0} \Gamma_{m_{1}, 2^{j z}}^{j, 0}\left\{(-1)^{n}\right. \\
& \cdot v\left(\frac{k_{0}}{2^{j_{x}}}, \frac{l_{0}}{2^{j_{y}}}, 1, t\right) s_{2 m}\left(\frac{k_{0}}{2^{j_{x}}}, \frac{l_{0}}{2^{j_{y}}}, t\right) \\
& -\frac{d s_{2 n}\left(k_{0} / 2^{j_{x}}, l_{0} / 2^{j_{y}}, t\right)}{d t}+(-1)^{n} \\
& \cdot f\left[s_{2 n}^{2}\left(\frac{k_{0}}{2^{j_{x}}}, \frac{l_{0}}{2^{j_{y}}}, t\right)+s_{2 m}^{2}\left(\frac{k_{0}}{2^{j_{x}}}, \frac{l_{0}}{2^{j_{y}}}, t\right)\right] \\
& \cdot s_{2 m}\left(\frac{k_{0}}{2^{j_{x}}}, \frac{l_{0}}{2^{j_{y}}}, t\right) \\
& -g\left[s_{2 n}^{2}\left(\frac{k_{0}}{2^{j_{x}}}, \frac{l_{0}}{2^{j_{y}}}, t\right)+s_{2 m}^{2}\left(\frac{k_{0}}{2^{j_{x}}}, \frac{l_{0}}{2^{j_{y}}}, t\right)\right] \\
& \left.\cdot s_{2 n}\left(\frac{k_{0}}{2^{j_{x}}}, \frac{l_{0}}{2^{j_{y}}}, t\right)+(-1)^{n} h_{m}\left(\frac{k_{0}}{2^{j_{x}}}, \frac{l_{0}}{2^{j_{y}}}, 1, t\right)\right\} \text {. }
\end{aligned}
$$

Note that in the semidiscretization system (15) of the three-dimensional generalized nonlinear Schrödinger equation (1), both of $\mathbf{A}$ and $\mathbf{B}$ are constant matrices, which are completely independent of the unknown vector $\mathbf{U}(t)$ and time $t$. Thus, in subsequent time integration for solving nonlinear ordinary differential equations (15), no matrix generated from spatial discretization needs updating, implying that full decoupling between spatial and temporal discretizations is achieved in the present wavelet algorithm.

Since the Coiflet scaling function $\phi(x)$ holds the orthogonality, following definition (5) of wavelet basis $\varphi_{j, k}(x)$, one can obtain the relation $\Gamma_{l, k}^{j, 0}=\int_{0}^{1} \varphi_{j, l}(x) \varphi_{j, k}(x) d x=2^{-j} \delta_{l, k}$ for $8 \leq l \leq 2^{j}-10$ or $8 \leq k \leq 2^{j}-10$. Therefore, when the decomposition level $j \geq 5$, the symmetrical matrix $\mathbf{A}$ is almost a diagonal matrix with only $\left(2^{j}+113\right)^{3}$ nonzero elements located in $\left(2^{j}-1\right)^{6}$ positions, whose distribution pattern is shown in Figure 1 when $j=6$. Thus, the computational cost for estimating the inverse matrix $\mathbf{A}^{-1}$ of matrix $\mathbf{A}$ is not very high. Moreover, considering the Coiflet scaling function $\phi(x)$ with the compact support $[0,17]$, there is the relation $\Gamma_{l, k}^{j, 2}=0$ for $|l-k| \geq 17$. Therefore, $\mathbf{B}$ is a banded matrix with bandwidth $b=33^{3}$ for the decomposition level $j \geq 5$. Therefore, the computational complexity of the proposed wavelet method is acceptable.

Finally, one can obtain an approximate solution of GNSE (1) by employing a time integration scheme to solve the nonlinear ordinary differential equations (15). In this study, 


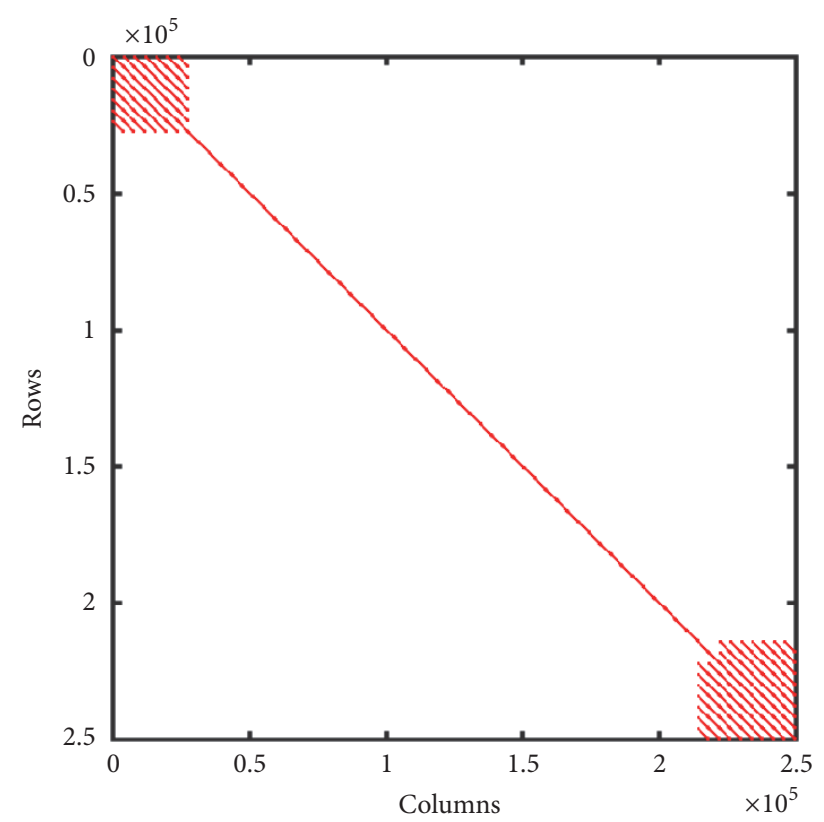

FIgURE 1: The sparsity pattern of matrix $\mathbf{A}$ for $j=6$. the classical fourth-order explicit Runge-Kutta method is adopted, and then we have

$$
\begin{aligned}
\mathbf{K}_{1} & =\Delta t \mathbf{G}\left[\mathbf{U}\left(t_{\bar{n}}\right), t_{\bar{n}}\right], \\
\mathbf{K}_{2} & =\Delta t \mathbf{G}\left[\mathbf{U}\left(t_{\bar{n}}\right)+\frac{\mathbf{K}_{1}}{2}, t_{\bar{n}+1 / 2}\right], \\
\mathbf{K}_{3} & =\Delta t \mathbf{G}\left[\mathbf{U}\left(t_{\bar{n}}\right)+\frac{\mathbf{K}_{2}}{2}, t_{\bar{n}+1 / 2}\right], \\
\mathbf{K}_{4} & =\Delta t \mathbf{G}\left[\mathbf{U}\left(t_{\bar{n}}\right)+\mathbf{K}_{3}, t_{\bar{n}+1}\right], \\
\mathbf{U}\left(t_{\bar{n}+1}\right) & =\mathbf{U}\left(t_{\bar{n}}\right)+\frac{\mathbf{K}_{1}+2 \mathbf{K}_{2}+2 \mathbf{K}_{3}+\mathbf{K}_{4}}{6}
\end{aligned}
$$

in which $t_{\bar{n}}=\bar{n} \Delta t$, and $\Delta t$ is the time step. Iteratively applying (19) and directly using the initial condition (2), one can obtain the unknown vector $\mathbf{U}\left(t_{\bar{n}}\right)=\left\{\mathbf{U}_{1}\left(t_{\bar{n}}\right), \mathbf{U}_{2}\left(t_{\bar{n}}\right)\right\}^{\mathrm{T}}$ at each time step, which can be used to reconstruct the approximate solution $\psi(\mathbf{x}, t)$ based on scheme (10).

\section{Numerical Examples}

In this section, we use several numerical examples to demonstrate the accuracy and applicability of the proposed wavelet method for solving two- and three-dimensional generalized nonlinear Schrödinger equations (1).

To effectively evaluate the performance of numerical solutions, we introduce the error norms $E_{\max }$ and $E_{2}$, and the

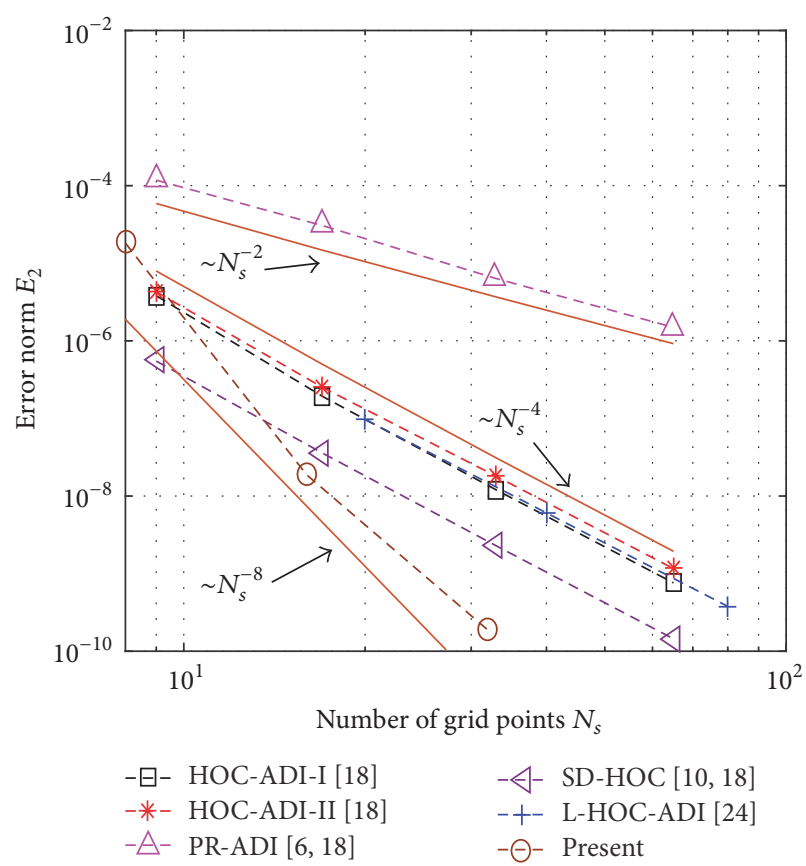

FIGURE 2: Error norm $E_{2}$ of numerical solutions at $t=1$ with $\Delta t=$ 0.0001 for various $N_{s}$ for Example 1 .

corresponding convergence rates in space $R_{\max }$ and $R_{2}$, which are, respectively, defined by $[9,18,20,23]$

$$
\begin{aligned}
E_{\max } & =\max _{k}\left\{\left|\psi_{\text {num }}\left(\mathbf{x}_{k}, t\right)-\psi_{\text {exact }}\left(\mathbf{x}_{k}, t\right)\right|\right\}, \\
E_{2} & =\sqrt{\frac{\sum_{k=1}^{N}\left|\psi_{\text {num }}\left(\mathbf{x}_{k}, t\right)-\psi_{\text {exact }}\left(\mathbf{x}_{k}, t\right)\right|^{2}}{N_{s}}}, \\
R_{\max (2)} & =\frac{\ln \left[E_{\max (2)}\left(N_{s 1}\right)\right]-\ln \left[E_{\max (2)}\left(N_{s 2}\right)\right]}{\ln \left(N_{s 2} / N_{s 1}\right)}
\end{aligned}
$$

in which $\psi_{\text {num }}$ and $\psi_{\text {exact }}$ are the numerical and exact solutions, respectively, and $N_{s}=2^{j}$ is the number of grid points in space. To make the convergence rates $R_{\max }$ and $R_{2}$ estimated by (22) being spatial convergence rates, a time step $\Delta t$ small enough to guarantee that the errors mainly come from the spatial discretization is adopted in the following computation.

Example 1. Consider the two-dimensional linear Schrödinger equation with trapping potential $[6,10,18,24]$

$$
\begin{array}{r}
\mathrm{i} \frac{\partial \psi}{\partial t}+\nabla^{2} \psi+\left[3-2 \tanh ^{2}(x)-2 \tanh ^{2}(y)\right] \psi=0, \\
\mathbf{x} \in[0,1]^{2}, t \geq 0
\end{array}
$$

with the initial and boundary conditions extracted from its exact solution $\psi(x, y, t)=\operatorname{iexp}(\mathrm{it}) /[\cosh (x) \cosh (y)]$. Figure 2 shows the error norm $E_{2}$ of the present wavelet solution at time $t=1$ under $\Delta t=10^{-4}$ for various numbers of grid points in space $N_{s}$, as well as those obtained by using the 
TABLE 1: Error norm $E_{2}$ of numerical solutions at $t=1$ with $\Delta t=$ $1 / 25000$ for Example 2.

\begin{tabular}{lcccc}
\hline \multirow{2}{*}{$N_{s}$} & \multicolumn{2}{c}{ Present } & \multicolumn{2}{c}{ HOC-ADI [34] } \\
& $E_{2}$ & $R_{2}$ & $E_{2}$ & $R_{2}$ \\
\hline 8 & $7.10 E-03$ & - & $1.13 E-03$ & - \\
16 & $1.95 E-05$ & 8.51 & $7.38 E-05$ & 3.94 \\
32 & $2.02 E-07$ & 6.59 & $4.74 E-06$ & 3.96 \\
64 & $3.04 E-09$ & 6.06 & $3.00 E-07$ & 3.98 \\
\hline
\end{tabular}

Peaceman-Rachford alternating direction implicit method (PR-ADI) [6, 18], semidiscrete high-order compact scheme (SD-HOC) [10, 18], high-order compact alternating direction implicit method (HOC-ADI) [18], and the linearized compact alternating direction implicit method (L-HOC-ADI) [24].

Example 2. We consider the two-dimensional cubic nonlinear Schrödinger equation [34]

$$
\mathrm{i} \frac{\partial \psi}{\partial t}+\frac{1}{2} \nabla^{2} \psi+\psi-|\psi|^{2} \psi=0, \quad \mathbf{x} \in[0,2 \pi]^{2}, t \geq 0
$$

subjected to the initial and boundary conditions extracted from the exact solution $\psi(x, y, t)=\exp [\mathrm{i}(x+y-t)]$. In Table 1, we list the error norms $E_{2}$ of numerical solutions at time $t=1$ under $\Delta t=1 / 25000$ achieved, respectively, from the proposed wavelet method, and the higher order compact alternating direction implicit method (HOC-ADI) [34].

Example 3. Consider the two-dimensional Gross-Pitaevskii Equation [28]

$$
\mathrm{i} \frac{\partial \psi}{\partial t}+\nabla^{2} \psi+\beta|\psi|^{2} \psi=0, \quad \mathbf{x} \in[0,2 \pi]^{2}, t \geq 0
$$

with $\beta=2$, and the initial and Dirichlet boundary conditions extracted from the exact solution $\psi(x, y, t)=\exp \{\mathrm{i}[x+y-$ $(2-\beta) t]\}$. Figure 3 shows the error norm $E_{\max }$ of numerical solutions at time $t=1$ under $\Delta t=0.001$ obtained by using the present wavelet method, the fourth-order nonlinear compact and energy conservative difference scheme (FONCECD) [28], and the fourth-order linearized compact and energy conservative difference scheme (FOLCECD) [28].

Example 4. We consider the two-dimensional nonlinear Schrödinger equation $[9,23,24,34]$.

$$
\begin{aligned}
& \mathrm{i} \frac{\partial \psi}{\partial t}+\frac{1}{2} \nabla^{2} \psi-\left[1-\sin ^{2}(x) \sin ^{2}(y)\right] \psi-|\psi|^{2} \psi=0, \\
& \mathbf{x} \in[0,2 \pi]^{2}, t \geq 0 \\
& \psi(0, y, t)=\psi(2 \pi, y, t)=\psi(x, 0, t)=\psi(x, 2 \pi, t) \\
& =0 \text {, } \\
& \psi(x, y, 0)=\sin (x) \sin (y)
\end{aligned}
$$

which has the exact solution $\psi(x, y, t)=$ $\sin (x) \sin (y) \exp (-2 \mathrm{i} t)$. Figure 4 plots the error norms of the present wavelet solutions with $\Delta t=1 / 5000$ as a function

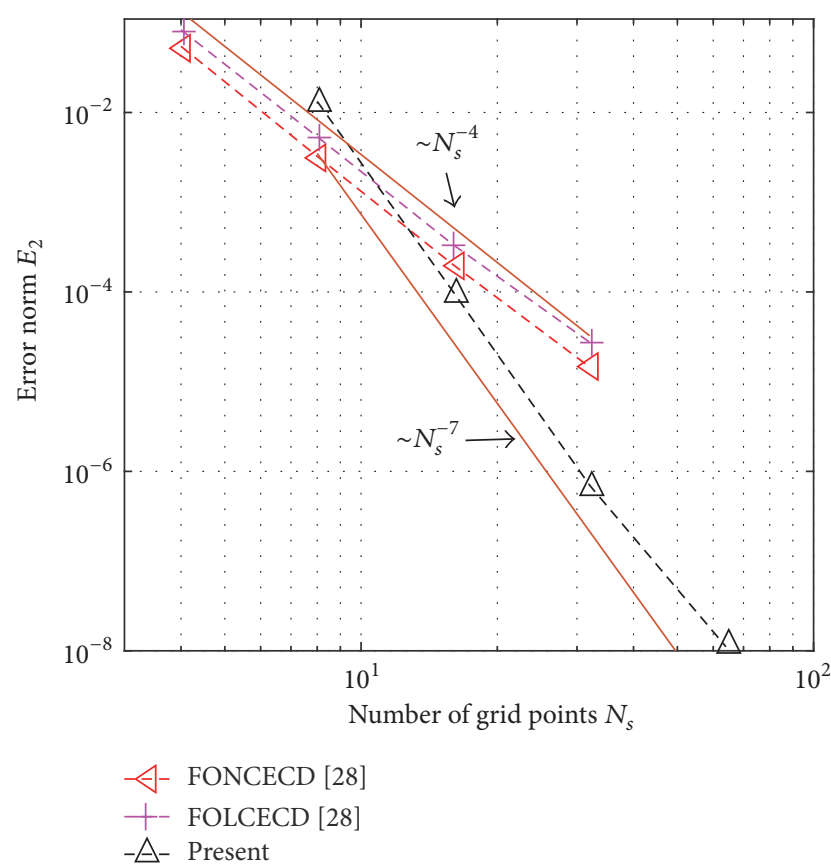

FIGURE 3: Error norm $E_{\max }$ of numerical solutions at $t=1$ with $\Delta t=0.001$ for various $N_{s}$ for Example 3 .

of the number of grid points in space $N_{s}$, as well as those obtained by using the split-step finite difference method (SSFD) [9], HOC-ADI [23, 34], linearized alternating direction implicit scheme (L-ADI) [24], L-HOC-ADI [24], and CCD-PRADI [34].

Example 5. Consider the two-dimensional nonlinear Schrödinger equation with damping

$$
\begin{aligned}
\mathrm{i} \frac{\partial \psi}{\partial t}+\frac{1}{2} \nabla^{2} \psi+\left(1+e^{-2 t}\right) \psi-|\psi|^{2} \psi+\mathrm{i} \psi & =0, \\
\mathbf{x} & \in[0,2 \pi]^{2}, t \geq 0
\end{aligned}
$$

subjected to the initial and boundary conditions extracted from the exact solution $\psi(x, y, t)=\exp [\mathrm{i}(x+y)-t]$. The error norms of the present wavelet solutions at time $t=1$ and 3 under various space-time meshes are listed in Table 2.

Example 6. Consider the following two-dimensional nonlinear Schrödinger

$$
\begin{aligned}
\mathrm{i} \frac{\partial \psi}{\partial t}+ & \frac{1}{2} \nabla^{2} \psi+\left[|\psi|^{4}-|\psi|^{2}\right] \psi \\
+\left[-\sin ^{4}(x+y)+\sin ^{2}(x+y)\right] \psi & =0, \\
& \mathbf{x} \in[0, \pi]^{2}, t \geq 0
\end{aligned}
$$

with the initial condition and boundary condition extracted from the exact solution $\psi(x, y, t)=\sin (x+y) \exp (-\mathrm{i} t)$. Table 3 shows the spatial convergence of the present wavelet solutions at time $t=1$ and 3 . 
TABLE 2: Error norms of wavelet solutions at $t=1$ and 3 with $\Delta t=0.001$ for Example 5.

\begin{tabular}{|c|c|c|c|c|c|c|c|c|}
\hline \multirow{2}{*}{$N_{s}$} & \multicolumn{4}{|c|}{$t=1$} & \multicolumn{4}{|c|}{$t=3$} \\
\hline & $E_{\max }$ & $R_{\max }$ & $E_{2}$ & $R_{2}$ & $E_{\max }$ & $R_{\max }$ & $E_{2}$ & $R_{2}$ \\
\hline 8 & $5.82 e-03$ & - & $2.94 e-03$ & - & $8.63 e-04$ & - & $4.35 e-04$ & - \\
\hline 16 & $2.59 e-05$ & 7.81 & $9.01 e-06$ & 8.35 & $3.84 e-06$ & 7.81 & $1.39 e-06$ & 8.29 \\
\hline 32 & $2.37 e-07$ & 6.78 & $8.42 e-08$ & 6.74 & $7.54 e-08$ & 5.67 & $1.56 e-08$ & 6.47 \\
\hline 64 & $3.82 e-09$ & 5.95 & $1.04 e-09$ & 6.34 & $6.05 e-10$ & 6.96 & $1.56 e-10$ & 6.65 \\
\hline
\end{tabular}

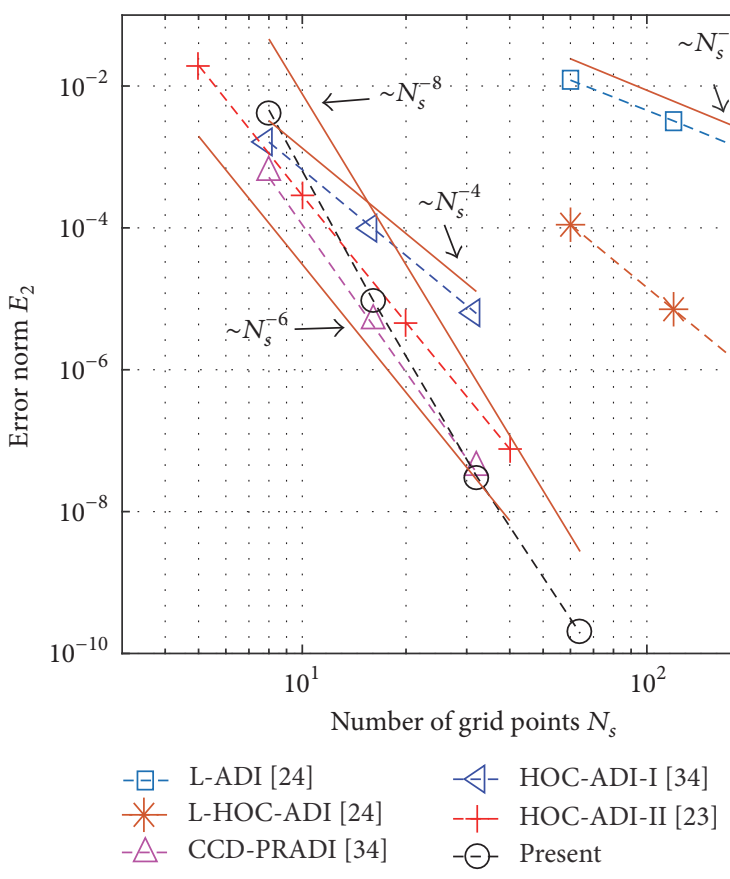

(a)

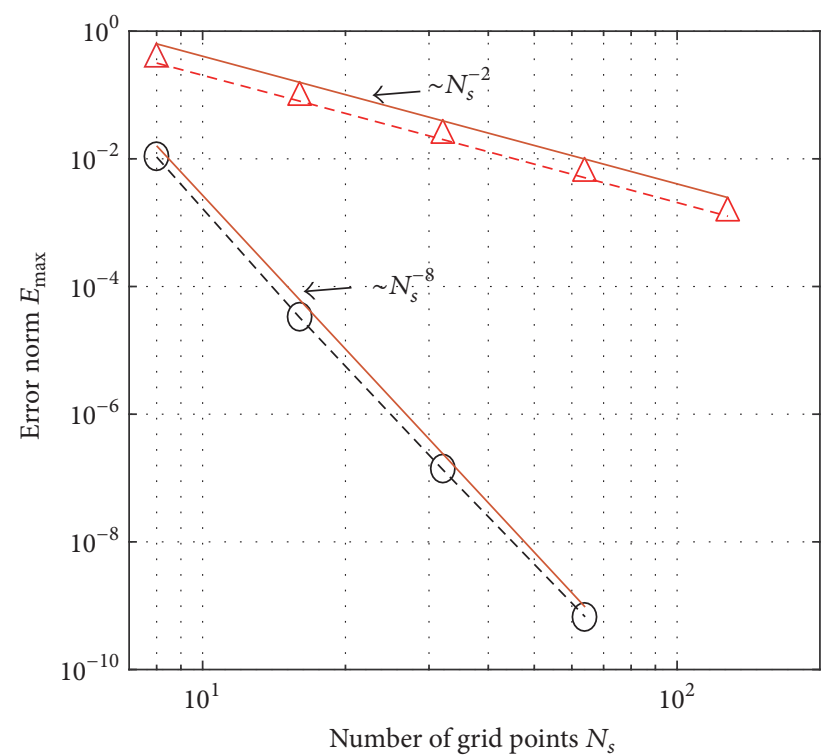

- - SSFD [9]

$-\odot$ - Present

(b)

FIGURE 4: Error norm of numerical solutions at $t=1$ (a) and 2 (b) with $\Delta t=0.0002$ for various $N_{s}$ for Example 4.

Example 7. We consider the three-dimensional nonlinear Schrödinger equation [9].

$$
\begin{aligned}
& \mathrm{i} \frac{\partial \psi}{\partial t}+\frac{1}{2} \nabla^{2} \psi-\left[1-\sin ^{2}(x) \sin ^{2}(y) \sin ^{2}(z)\right] \psi \\
& \quad-|\psi|^{2} \psi=0, \quad \mathbf{x} \in[0,2 \pi]^{3}, t \geq 0, \\
& \psi(0, y, z, t)=\psi(2 \pi, y, z, t)=\psi(x, 0, z, t) \\
& \quad=\psi(x, 2 \pi, z, t)=0, \\
& \psi(x, y, 0, t)=\psi(x, y, 2 \pi, t)=0, \\
& \psi(x, y, z, 0)=\sin (x) \sin (y) \sin (z)
\end{aligned}
$$

whose exact solution is $\psi(x, y, z, t)=$ $\sin (x) \sin (y) \sin (z) \exp (-\mathrm{i} 5 t / 2)$. In Table 4 , we make a comparison of the error norm $E_{\max }$ between the present wavelet solutions and those achieved from SSFD [9].

It can be seen from Tables 1-4 and Figures 2-4 that the proposed space-time fully decoupled wavelet Galerkin method has a good accuracy, efficiency, and stability. Moreover, these results also demonstrate that its order of convergence in space can go up to 6 . In addition, we can see from the comparisons shown in Figures 2-4 and Tables 1-4 that when a relatively fine mesh is adopted, the present wavelet solutions have a much better numerical accuracy than those obtained by many other existing numerical methods, including PRADI $[6,18]$, SD-HOC $[10,18]$, HOC-ADI $[18,23,34]$, L-HOCADI [24], FONCECD [28], FOLCECD [28], SSFD [9], and L-ADI [24].

\section{Conclusion}

In this study, we introduced a sampling approximation for a function defined on a three-dimensional domain by combining Coiflet-type wavelet expansion and technique of boundary extension. On the basis of such a wavelet approximation, a space-time fully decoupled wavelet Galerkin is proposed to solve uniformly the multidimensional generalized nonlinear Schrödinger equation with damping, in which all matrices generated from the spatial discretization of nonlinear partial differential equations are completely independent of time 
TABLE 3: Error norms of wavelet solutions at $t=1$ and 3 with $\Delta t=0.0005$ for Example 6 .

\begin{tabular}{lcccccccr}
\hline$N_{s}$ & \multicolumn{2}{c}{$t=1$} & & \multicolumn{2}{c}{$t=3$} & $E_{2}$ & $R_{2}$ \\
\hline 8 & $E_{\max }$ & $R_{\max }$ & $E_{2}$ & $R_{2}$ & $E_{\max }$ & $R_{\max }$ & $7.71 e-04$ & - \\
16 & $6.84 e-04$ & - & $3.70 e-04$ & - & $1.32 e-03$ & - & 9.03 \\
32 & $1.20 e-06$ & 9.15 & $4.82 e-07$ & 9.58 & $3.59 e-06$ & 8.52 & $1.48 e-06$ \\
64 & $1.43 e-08$ & 6.39 & $7.02 e-09$ & 6.10 & $4.74 e-08$ & 6.24 & $2.22 e-08$ & 6.06 \\
\hline
\end{tabular}

TABLE 4: Error norm $E_{\max }$ and convergence rate $R_{\max }$ of numerical solutions at $t=2$ for Example 7 .

\begin{tabular}{lcccc}
\hline \multirow{2}{*}{$N_{s}$} & \multicolumn{2}{c}{ Present $(\Delta t=0.0002)$} & \multicolumn{2}{c}{$\operatorname{SSFD}(\Delta t=0.0001)[9]$} \\
& $E_{\max }$ & $R_{\max }$ & $E_{\max }$ & $R_{\max }$ \\
\hline 8 & $1.28 E-02$ & - & $8.40 E-01$ & - \\
16 & $4.56 E-05$ & 8.13 & $2.13 E-01$ & 1.98 \\
32 & $1.44 E-07$ & 8.31 & $5.36 E-02$ & 1.99 \\
64 & - & - & $1.34 E-02$ & 2.00 \\
128 & - & - & $3.35 E-03$ & 2.00 \\
\hline
\end{tabular}

and need not be updated in the time integration. By solving seven widely considered test problems, the present wavelet algorithm shows considerably high precision and fast convergence rate in space compared with many other existing numerical methods.

\section{Conflicts of Interest}

The authors declare that they have no conflicts of interest.

\section{Acknowledgments}

This research is supported by grants from the National Natural Science Foundation of China (11502103, 11421062), the Fundamental Research Funds for the Central Universities (lzujbky-2015-178), and the Open Fund of State Key Laboratory of Structural Analysis for Industrial Equipment (GZ15115).

\section{References}

[1] Y. S. Kivshar and B. A. Malomed, "Dynamics of solitons in nearly integrable systems," Reviews of Modern Physics, vol. 61, no. 4, pp. 763-915, 1989.

[2] F. Dalfovo, S. Giorgini, L. P. Pitaevskii, and S. Stringari, "Theory of Bose-Einstein condensation in trapped gases," Reviews of Modern Physics, vol. 71, no. 3, pp. 463-512, 1999.

[3] G. P. Agrawal, Nonlinear Fiber Optics, Academic Press, San Diego, Calif, USA, 4th edition, 2007.

[4] D. R. Solli, C. Ropers, P. Koonath, and B. Jalali, "Optical rogue waves," Nature, vol. 450, no. 7172, pp. 1054-1057, 2007.

[5] Y. Ye, "Self-similar solutions for nonlinear Schrödinger equations," Mathematical Problems in Engineering, vol. 2009, Article ID 298980, 15 pages, 2009.

[6] M. Subaşi, "On the finite-differences schemes for the numerical solution of two dimensional Schrödinger equation," Numerical
Methods for Partial Differential Equations, vol. 18, no. 6, pp. 752758, 2002.

[7] W. Bao and D. Jaksch, "An explicit unconditionally stable numerical method for solving damped nonlinear Schrödinger equations with a focusing nonlinearity," SIAM Journal on Numerical Analysis, vol. 41, no. 4, pp. 1406-1426, 2003.

[8] W. Bao, D. Jaksch, and P. A. Markowich, "Numerical solution of the Gross-Pitaevskii equation for Bose-Einstein condensation," Journal of Computational Physics, vol. 187, no. 1, pp. 318-342, 2003.

[9] H. Wang, "Numerical studies on the split-step finite difference method for nonlinear Schrödinger equations," Applied Mathematics and Computation, vol. 170, no. 1, pp. 17-35, 2005.

[10] J. C. Kalita, P. Chhabra, and S. Kumar, "A semi-discrete higher order compact scheme for the unsteady two-dimensional Schrödinger equation," Journal of Computational and Applied Mathematics, vol. 197, no. 1, pp. 141-149, 2006.

[11] M. Dehghan and A. Shokri, "A numerical method for twodimensional Schrödinger equation using collocation and radial basis functions," Computers \& Mathematics with Applications, vol. 54, no. 1, pp. 136-146, 2007.

[12] N. H. Sweilam, "Variational iteration method for solving cubic nonlinear Schrödinger equation," Journal of Computational and Applied Mathematics, vol. 207, no. 1, pp. 155-163, 2007.

[13] M. Dehghan and D. Mirzaei, "Numerical solution to the unsteady two-dimensional Schrödinger equation using meshless local boundary integral equation method," International Journal for Numerical Methods in Engineering, vol. 76, no. 4, pp. 501-520, 2008.

[14] A. K. Alomari, M. S. Noorani, and R. Nazar, "Explicit series solutions of some linear and nonlinear Schrodinger equations via the homotopy analysis method," Communications in Nonlinear Science and Numerical Simulation, vol. 14, no. 4, pp. 11961207, 2009.

[15] A. Mohebbi and M. Dehghan, "The use of compact boundary value method for the solution of two-dimensional Schrödinger equation," Journal of Computational and Applied Mathematics, vol. 225, no. 1, pp. 124-134, 2009.

[16] H.-L. Liao, Z.-Z. Sun, and H.-S. Shi, "Error estimate of fourthorder compact scheme for linear Schrödinger equations," SIAM Journal on Numerical Analysis, vol. 47, no. 6, pp. 4381-4401, 2010.

[17] F. G. Mertens, N. R. Quintero, and A. R. Bishop, "Nonlinear Schrödinger equation with spatiotemporal perturbations," Physical Review E: Statistical, Nonlinear, and Soft Matter Physics, vol. 81, no. 1, Article ID 016608, 2010.

[18] Z. F. Tian and P. X. Yu, "High-order compact ADI (HOCADI) method for solving unsteady 2D Schrödinger equation," Computer Physics Communications, vol. 181, no. 5, pp. 861-868, 2010. 
[19] Z. Gao and S. Xie, "Fourth-order alternating direction implicit compact finite difference schemes for two-dimensional Schrödinger equations," Applied Numerical Mathematics, vol. 61, no. 4, pp. 593-614, 2011.

[20] D. He and K. Pan, "An unconditionally stable linearized CCD-ADI method for generalized nonlinear Schrödinger equations with variable coefficients in two and three dimensions," Computers \& Mathematics with Applications, vol. 73, no. 11, pp. 2360-2374, 2017.

[21] S. Wang and L. Zhang, "Split-step orthogonal spline collocation methods for nonlinear Schrödinger equations in one, two, and three dimensions," Applied Mathematics and Computation, vol. 218, no. 5, pp. 1903-1916, 2011.

[22] H. Zhu, Y. Chen, S. Song, and H. Hu, "Symplectic and multisymplectic wavelet collocation methods for two-dimensional Schrödinger equations," Applied Numerical Mathematics, vol. 61, no. 3, pp. 308-321, 2011.

[23] L. Kong, Y. Duan, L. Wang, X. Yin, and Y. Ma, "Spectrallike resolution compact ADI finite difference method for the multi-dimensional Schrödinger equations," Mathematical and Computer Modelling, vol. 55, no. 5-6, pp. 1798-1812, 2012.

[24] Y. Xu and L. Zhang, "Alternating direction implicit method for solving two-dimensional cubic nonlinear Schrödinger equation," Computer Physics Communications, vol. 183, no. 5, pp. 1082-1093, 2012.

[25] S. Abbasbandy, H. Roohani Ghehsareh, and I. Hashim, "A meshfree method for the solution of two-dimensional cubic nonlinear Schrödinger equation," Engineering Analysis with Boundary Elements, vol. 37, no. 6, pp. 885-898, 2013.

[26] X. Antoine, W. Bao, and C. Besse, "Computational methods for the dynamics of the nonlinear Schrödinger/Gross-Pitaevskii equations," Computer Physics Communications, vol. 184, no. 12, pp. 2621-2633, 2013.

[27] M. Dehghan and F. Emami-Naeini, "The Sinc-collocation and Sinc-Galerkin methods for solving the two-dimensional Schrödinger equation with nonhomogeneous boundary conditions," Applied Mathematical Modelling, vol. 37, no. 22, pp. 93799397, 2013.

[28] T. Wang, B. Guo, and Q. Xu, "Fourth-order compact and energy conservative difference schemes for the nonlinear Schrödinger equation in two dimensions," Journal of Computational Physics, vol. 243, pp. 382-399, 2013.

[29] A. Taleei and M. Dehghan, "Time-splitting pseudo-spectral domain decomposition method for the soliton solutions of the one- and multi-dimensional nonlinear Schrödinger equations," Computer Physics Communications, vol. 185, no. 6, pp. 15151528, 2014.

[30] W. C. Tay and E. L. Tan, "Pentadiagonal alternatingdirection-implicit finite-difference time-domain method for two-dimensional Schrödinger equation," Computer Physics Communications, vol. 185, no. 7, pp. 1886-1892, 2014.

[31] L. W. Zhang, Y. J. Deng, K. M. Liew, and Y. M. Cheng, "The improved complex variable element-free Galerkin method for two-dimensional Schrödinger equation," Computers \& Mathematics with Applications, vol. 68, no. 10, pp. 1093-1106, 2014.

[32] L. W. Zhang and K. M. Liew, "An element-free based solution for nonlinear Schrödinger equations using the ICVMLS-Ritz method," Applied Mathematics and Computation, vol. 249, pp. 333-345, 2014.

[33] A. H. Bhrawy and M. A. Abdelkawy, "A fully spectral collocation approximation for multi-dimensional fractional
Schrödinger equations," Journal of Computational Physics, vol. 294, pp. 462-483, 2015.

[34] L. Z. Li, H.-W. Sun, and S.-C. Tam, "A spatial sixth-order alternating direction implicit method for two-dimensional cubic nonlinear Schrödinger equations," Computer Physics Communications, vol. 187, pp. 38-48, 2015.

[35] P. Mocz and S. Succi, "Numerical solution of the nonlinear Schrödinger equation using smoothed-particle hydrodynamics," Physical Review E: Statistical, Nonlinear, and Soft Matter Physics, vol. 91, no. 5, Article ID 053304, 2015.

[36] N. R. Quintero, F. G. Mertens, and A. R. Bishop, "Soliton stability criterion for generalized nonlinear Schrödinger equations," Physical Review E: Statistical, Nonlinear, and Soft Matter Physics, vol. 91, no. 1, Article ID 012905, 2015.

[37] T. W. Sheu and L. Lin, "Dispersion relation equation preserving FDTD method for nonlinear cubic Schrödinger equation," Journal of Computational Physics, vol. 299, pp. 1-21, 2015.

[38] X. Liu, J. Wang, and Y. Zhou, "A space-time fully decoupled wavelet Galerkin method for solving two-dimensional Burgers' equations," Computers \& Mathematics with Applications, vol. 72, no. 12, pp. 2908-2919, 2016.

[39] Y. Meyer, Wavelets and Operators, Cambridge University Press, Cambridge, UK, 1992.

[40] D. Roşca and J.-P. Antoine, "Locally supported orthogonal wavelet bases on the sphere via stereographic projection," Mathematical Problems in Engineering, vol. 2009, Article ID 124904, 13 pages, 2009.

[41] J. Wang, Generalized Theory and Arithmetic of Orthogonal Wavelets and Applications to Researches of Mechanics Including Piezoelectric Smart Structures, Lanzhou University, Lanzhou, China, 2001 (Chinese).

[42] X. Liu, A Wavelet Method for Uniformly Solving Nonlinear Problems and Its Application to Quantitative Research on Flexible Structures with Large Deformation, Lanzhou University, Lanzhou, China, 2014 (Chinese).

[43] X. Liu, Y. Zhou, L. Zhang, and J. Wang, "Wavelet solutions of Burgers' equation with high Reynolds numbers," Science China Technological Sciences, vol. 57, no. 7, pp. 1285-1292, 2014.

[44] X. Liu, J. Wang, and Y. Zhou, "A high-order accurate wavelet method for solving three-dimensional Poisson problems," Computer Modeling in Engineering \& Sciences, vol. 107, no. 6, pp. 433446, 2015. 


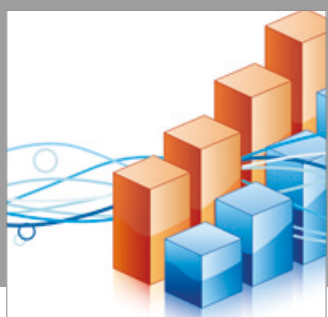

Advances in

Operations Research

vatersals

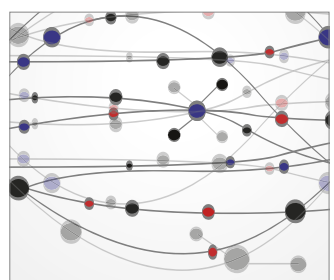

\section{The Scientific} World Journal
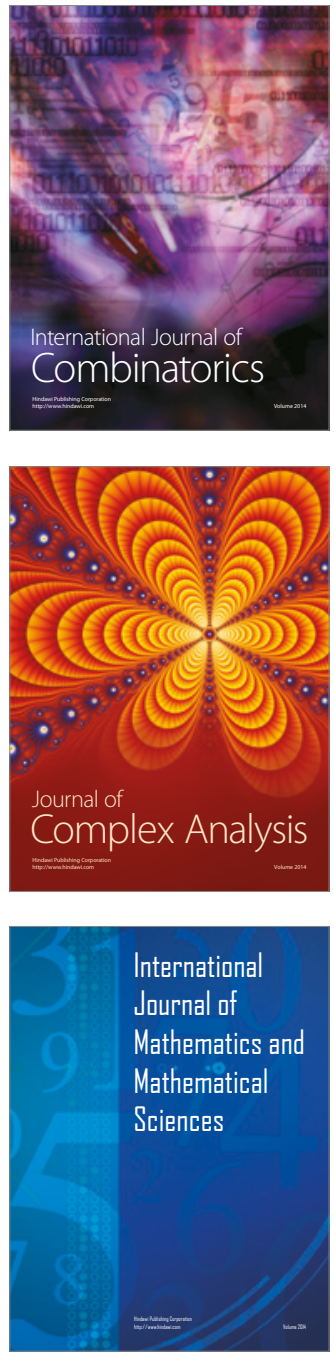
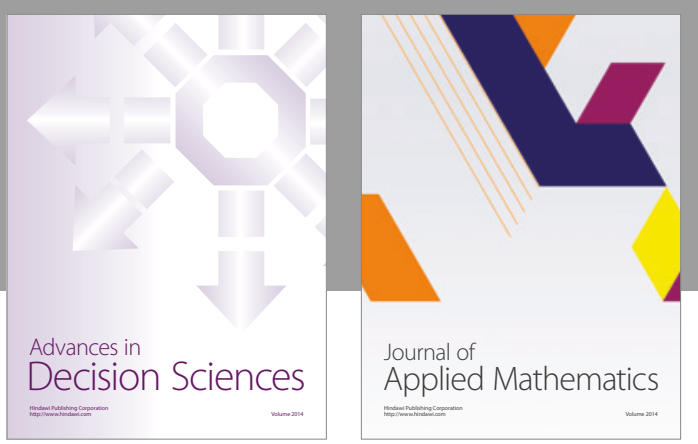

Algebra

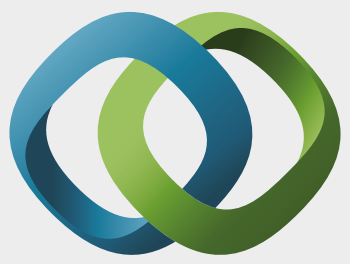

\section{Hindawi}

Submit your manuscripts at

https://www.hindawi.com
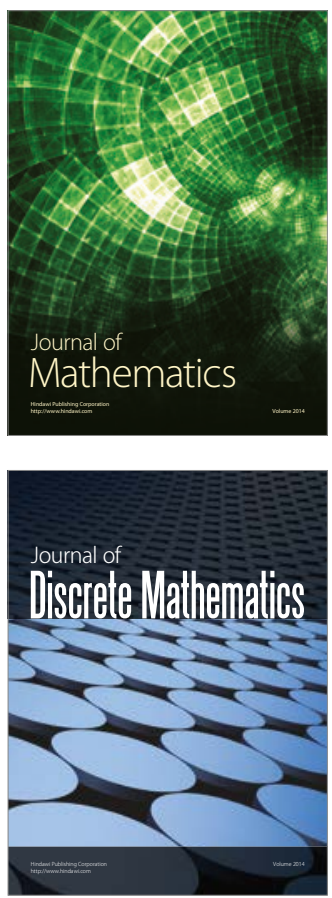

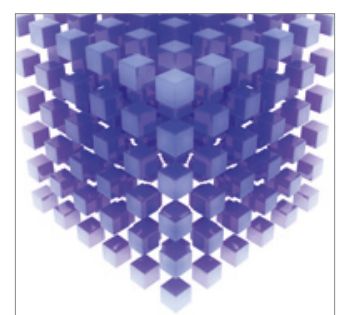

Mathematical Problems in Engineering
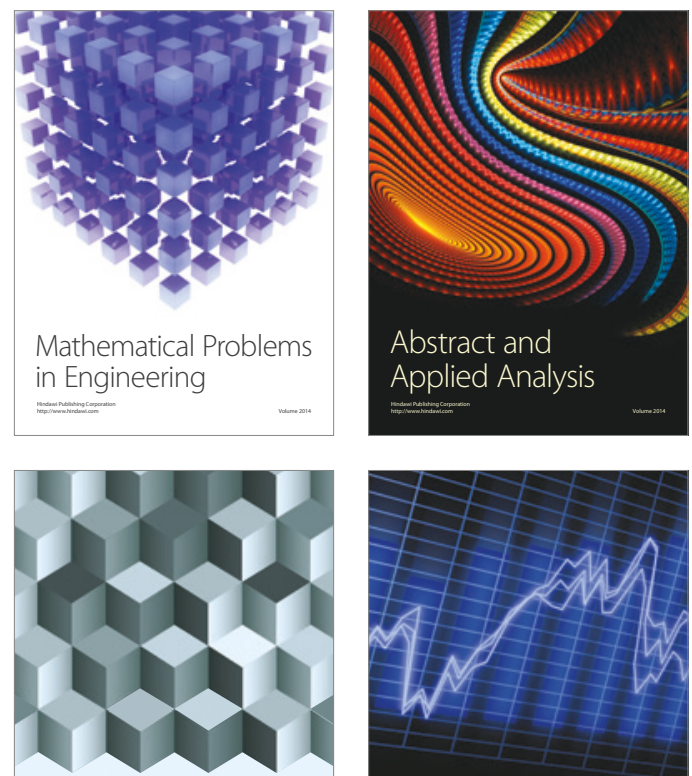

Journal of

Function Spaces

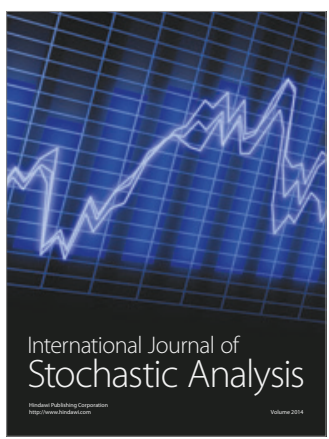

Probability and Statistics
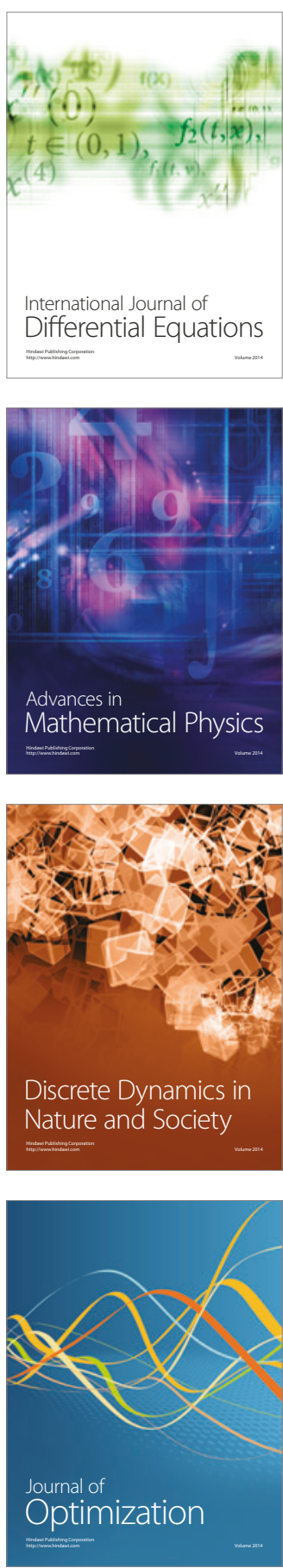